Journal of

Molecular Microbiology

and Biotechnology

\title{
Regression of Solid Tumors by Induction of MazF, a Bacterial mRNA Endoribonuclease
}

\author{
Tsutomu Shimazu $^{a}$ Oleg Mirochnitchenko ${ }^{b}$ Sangita Phadtare ${ }^{c}$ \\ Masayori Inouye $^{a}$ \\ a Department of Biochemistry and Molecular Biology, Robert Wood Johnson Medical School, Piscataway, N.J., \\ ${ }^{b}$ DCM/ORIP/Division of Program Coordination, Planning, and Strategic Initiatives Office of the Director, National \\ Institutes of Health (NIH), Bethesda, Md., and 'Department of Biomedical Sciences, Cooper Medical School of Rowan \\ University, Camden, N.J., USA
}

\section{Key Words}

MazF · Tumor regression $\cdot$ TA toxins

\begin{abstract}
MazF from Escherichia coli is an endoribonuclease that specifically cleaves mRNAs at ACA sequences. Its induction in mammalian cells has been shown to cause programmed cell death. Here we explored if a bacterial MazF-MazE toxin-antitoxin system can be used for gene therapy. For this, we first constructed a tetracycline-inducible MazF expression system in human embryonic kidney cells (T-Rex 293-mazF). Solid tumors were formed by injecting T-Rex 293-mazF cells into nude mice. All 8 mice injected with the cells developed solid tumors, which regressed upon induction of MazF. In 4 mice, tumors completely regressed, while in the remaining 4 mice, tumors reappeared after apparent significant regression, which was found to be due to the lack of presence of functional MazF. Notably, the MazF-mediated regression of the tumors was counteracted by the expression of its cognate antitoxin MazE. These results indicate that a bacterial MazFMazE toxin-antitoxin system may have potential to be used as a therapeutic tool.

(c) 2014 S. Karger AG, Basel
\end{abstract}

\section{KARGER}

E-Mail karger@karger.com

www.karger.com $/ \mathrm{mmb}$

\section{Introduction}

In tumor the cell death pathway such as apoptosis is commonly inactivated. Reactivation of this mechanism can thus be used to increase the effectiveness of anti-cancer therapy. Efforts to improve cancer therapy have focused on the development of more selective, biological mechanism-based targeted therapies that can overcome tumor resistance, as well as minimize toxic effects to normal cells. Cytotoxic ribonucleases (RNases) represent one such mechanism-based approach [Arnold and UlbrichHofmann, 2006; Benito et al., 2005; Leland and Raines, 2001; Wu et al., 1993]. These relatively small and highly cationic proteins selectively attack malignant cells, triggering apoptotic response by inhibition of protein synthesis [Ardelt et al., 2008; Leland and Raines, 2001]. These exhibit preferential toxicity towards cancer cells. Alternatively, the cellular levels of RNases and ribonuclease inhibitors in the cancer cells and their normal counterparts may be different. Thus, RNases represent a new therapeutic class of agents with broad clinical applications and new selective biologic activities. Among these enzymes, onconase (P30 protein, ranpirnase), bovine-seminal ribonu- 
clease and the Rana catesbeiana and Rana japonica lectins exert cytotoxic activity that is selective for tumor cells [Arnold and Ulbrich-Hofmann, 2006; Lee and Raines, 2008]. Onconase was first isolated from extracts of Rana pipiens eggs and early embryos that have potent in vivo anti-tumor activity [Ardelt et al., 2008; Costanzi et al., 2005]. When incubated with mammalian cells in culture, onconase shows cytotoxic and cytostatic activities. Onconase has been reported to suppress cancer cell growth in vitro, reduce tumor size in animals and increase the cytotoxicity of several chemotherapeutic agents [Costanzi et al., 2005; Lee and Shogen, 2008]. Cytotoxicity of RNases including onconase requires interaction of the RNases with the cell membrane leading to their internalization by endocytosis. RNases are then translocated to the cytosol where they cleave cellular RNA and block translation, resulting in apoptotic cell death [Lee and Shogen, 2008]. How efficiently a particular RNase carries out each of these steps determines its potency for cytotoxicity.

Bacterial cells also contain various RNases that function to regulate cell growth and cell death. In Escherichia coli, growth inhibition and subsequent cell death are controlled by unique genetic systems called the toxin-antitoxin (TA) systems. Most studied type II TA systems are composed of a pair of genes encoding two proteins, a stable toxin and an unstable antitoxin [Gerdes et al., 2005; Yamaguchi et al., 2011]. One of the TA modules on the E. coli genome, the mazEF system, consists of two adjacent genes, $m a z E$ and $m a z F$ [Aizenman et al., 1996]. MazF is a sequence-specific endoribonuclease that specifically cleaves at the ACA triplet sequence to block de novo protein synthesis, resulting in cell growth arrest and subsequent cell death [Inouye, 2006; Zhang et al., 2003]. The role of antitoxin MazE is to suppress the endoribonuclease activity of MazF and protect bacterial cells from cell death. Moreover, expression of MazF in mammalian cells causes dramatic mRNA degradation and inhibition of protein synthesis, leading to apoptotic cell death [Shima$\mathrm{zu}$ et al., 2007]. This MazF-mediated degradation of mRNA in mammalian cells led to apoptotic cell death. The co-expression of MazE with MazF completely inhibited MazF-induced mRNA degradation, inhibition of protein synthesis and apoptotic cell death in mammalian cells as well as in E. coli cells [Shimazu et al., 2007].

In this study, we demonstrate that sustained expression of wild-type MazF expression leads to dramatic reduction in the size of tumors in nude mice and the coexpression of antitoxin MazE with MazF counteracts the MazF-induced tumor regression.

\section{Results}

\section{MazF Induction Causes Regression of Tumors in}

Nude Mice

We have demonstrated that MazF induction in mammalian cells causes shutoff of de novo protein synthesis through mRNA degradation by cleavage at the ACA triplet sequences, resulting in NBK/BIK and BAKmediated apoptosis, while MazE antagonizes MazF-induced apoptosis [Shimazu et al., 2007]. In addition, it has been shown that onconase from $R$. pipiens induces apoptosis in mammalian cells, leading to tumor regression in nude mice [Lee and Shogen, 2008]. These findings led us to explore the effect of MazF on growth of human tumors in nude mice. To this end, a tetracycline (Tet)-inducible MazF expression system in human embryonic kidney cells (T-Rex 293) stably expressing the Tet repressor was developed through stable co-transfection of Tet-inducible MazF expression plasmid with pcDNA3 or the constitutive MazE expression plasmid [Shimazu et al., 2007]. RT-PCR with specific primers for the MazF- or MazE-coding region revealed Tet-dependent MazF mRNA expression and constitutive expression of MazE mRNA in established 293 cell lines (data not shown). The cell line 293 ( $m a z F / p c D N A 3$ ) was injected subcutaneously into nude mice and tumor growth was monitored over 45 days. MazF was induced in tumors in vivo in nude mice by administration of 100 $\mathrm{mg} / \mathrm{ml}$ of doxycycline (Dox) containing sterile water and the effect of MazF induction on tumors was assessed. As a control, tumor size was monitored in mice injected with 293 (MazF/pcDNA3) cells (MazFC\#1, \#2 and \#3) without Dox-mediated induction of MazF. As expected, in the control mice, all tumors increased in size up to 28 days at which point the experiment was terminated (fig. 1a). Effect of MazF induction was monitored independently in eight 293 ( $\mathrm{mazF} / \mathrm{pcDNA3}$ ) mice (MazF\#1-\#8). In 4 mice (MazF\#1, \#2, \#3 and \#4), the tumor size dramatically decreased in response to MazF induction, resulting in complete regression at 2 weeks, which was maintained even after a prolonged period of time (45 days) (fig. 1b). The upper panel in figure $2 \mathrm{a}$ shows a photographic example of tumor growth and MazF-mediated regression of the tumor (MazF\#1). Tumors in the remaining 4 mice (MazF\#5, \#6, \#7 and \#8) expressing MazF were also significantly decreased after MazF induction; however, tumor growth recurred within 2 weeks after MazF induction (fig. 1c). To check if the effect of MazF on tumor growth can be counteracted by its antitoxin MazE, the 293 

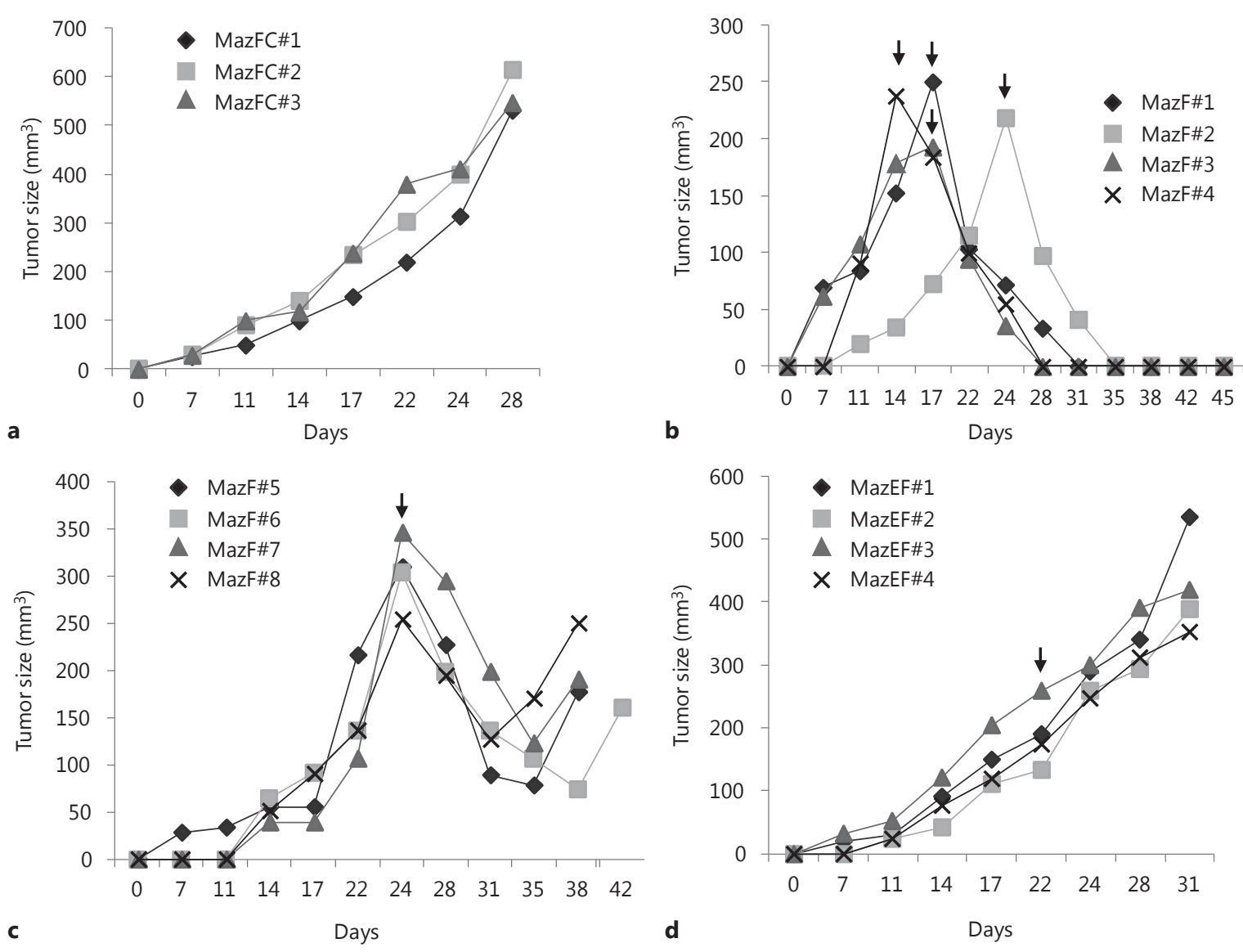

Fig. 1. Effect of MazF on tumors derived from transformed 293 cells in nude mice. a Tumor formation in nude mice without the induction of MazF: MazFC\#1-\#3. b Tumor formation in nude mice in which MazF is induced: MazF\#1-\#4. c Tumor formation in nude mice in which MazF is induced: MazF\#5-\#8. d Effect of co-expression of MazE with MazF on tumor formation in nude mice: MazEF\#1-\#4. Arrows indicate start point of MazF induction by administration of $100 \mathrm{mg} / \mathrm{ml}$ Dox; MazE is constitutively expressed in $\mathbf{d}$.

\section{Analysis of Lack of Continued Regression of Tumors} by MazF in Certain Nude Mice

As shown in figure 1c, tumor size in 4 out of 8 mice regressed for 10 days following MazF induction and then resumed growth. The lower panel in figure 2a shows a photographic example of tumor growth, MazF-mediated regression of the tumor to some extent followed by resumption of tumor growth (MazF\#5). This resumption of tumor growth may be due to the (i) absence of continued expression of MazF, (ii) acquired mutations in the mazF gene, or (iii) degradation of MazF. To analyze why tumor 


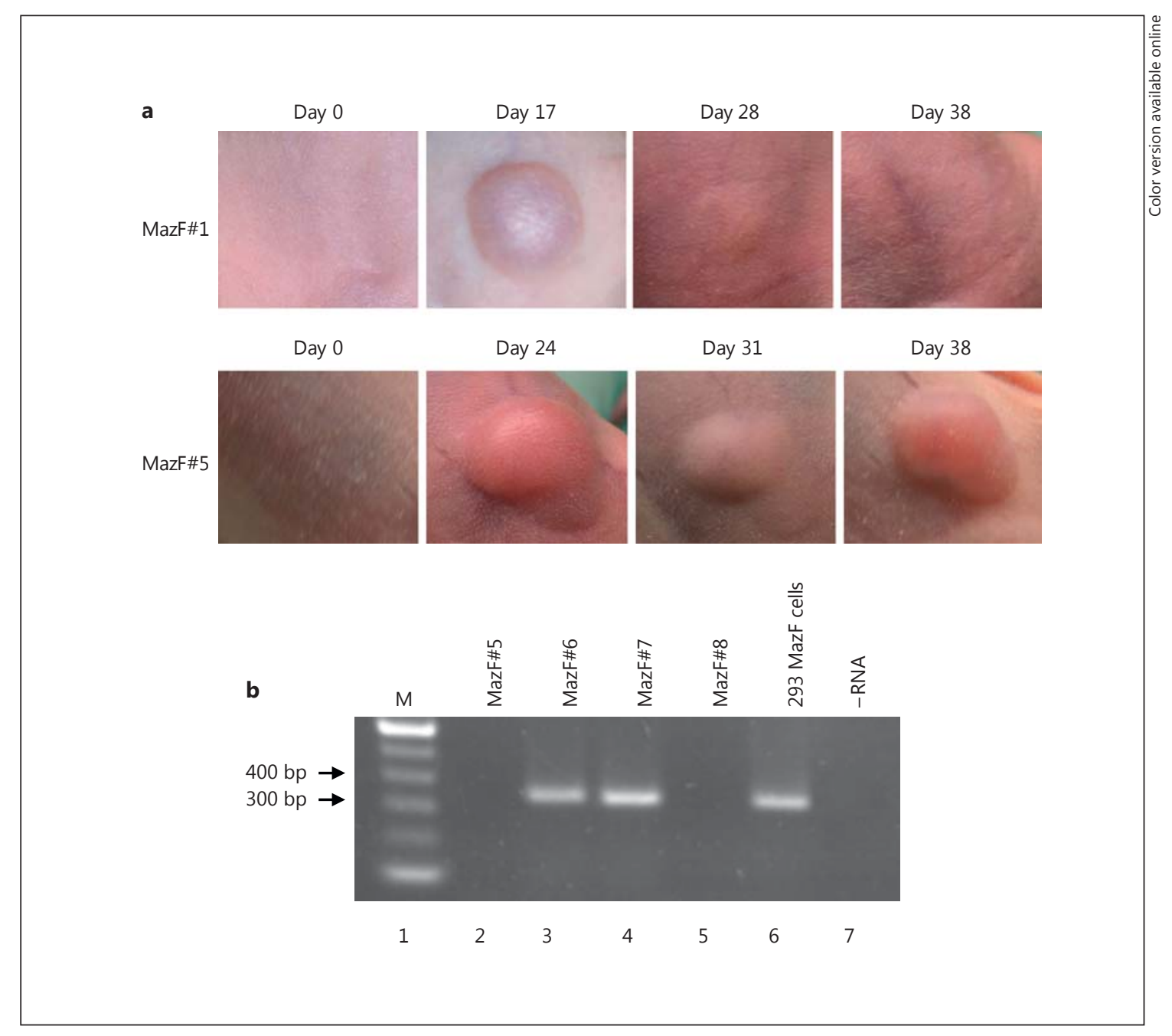

Fig. 2. a Visualization of the effect of MazF on tumors derived from transformed 293 cells in nude mice. Representative tumors from 293/mazF cells-injected mice (MazF\#1 and \#5) photographed at indicated time points. b MazF mRNA levels in tumors. RT-PCR was carried out using specific primers and total RNA isolated from each tumor as indicated. Lane 1: molecular weight markers were loaded is indicated as M. Lanes 6 and 7: control reactions with 293 MazF cells and without RNA, respectively.

re-growth occurred, the levels of MazF mRNA in 4 mice (MazF\#5, \#6, \#7 and \#8) were analyzed by RT-PCR using total RNA isolated from tumor from each dissected mice at the endpoint of monitoring tumor growth. Data revealed that in two tumors, MazF\#5 and \#8, MazF cDNA was not detected (fig. 2b; lanes 2 and 5). This suggested that MazF protein was either no longer expressed due to the degradation of mazF mRNA or that there may be mutations in the promoter region necessary to transcribe mazF mRNA. The 333-basepair MazF cDNA was clearly detected in MazF\#6 and MazF\#7 (fig. 2b; lanes 3 and 4), indicating that $293(\mathrm{mazF} / \mathrm{pcDNA} 3)$ cells in these two tu- mors still have capability to produce MazF mRNA at the endpoint of experiment. Next, DNA sequencing was carried out to check if mutations occurred in the coding region of MazF in these two tumors (MazF\#6 and MazF\#7). Indeed substitution mutations were detected for the lysine residues at 42nd and 56th positions (codon AA $\underline{\text { A }}$ ) in mazF mRNA from tumor MazF\#6 and MazF\#7, respectively. These lysine residues were changed to asparagine (codon: AAT). These mutations are not caused during PCR as sequencing of 10 independent plasmid clones with MazF cDNA amplified by PCR had the same mutations. Structural studies have suggested that K42 and K56 
amino acids are located in the domain of MazF that is important for its RNA-binding ability [Li et al., 2006]. This suggests that the K42 and K56 mutations cause the loss of MazF mRNA-binding activity and thus loss of its RNase activity in two tumors (MazF\#6 and MazF\#7), leading to recurrence of increased tumor size due to deficiency in apoptotic cell death.

\section{Discussion}

Degradation of mRNA and consequent shutoff of protein synthesis by RNases and RNA interference (RNAi) is a promising strategy for reducing tumor growth. We demonstrate here that MazF toxin, one of bacterial endoribonucleases, has potential to suppress tumor growth and to cause tumor regression. Interestingly, E. coli cells have several toxins to control cell growth by inhibition of protein synthesis through mRNA degradation and blocking translation elongation [Inouye, 2006; Yamaguchi et al., 2011]. Furthermore, various bacteria contain MazF homologues [Gerdes et al., 2005]. These bacterial toxins may also be tested for use as anti-cancer agents. Moreover, combination treatment with two different toxins, as well as with other synthetic anti-cancer drugs, may prove to be more effective. MazF is a small (111 amino acids) and basic protein (pI 8.3), similar to onconase that can be easily internalized into cells due to its biochemical characteristics, suggesting a possibility of direct treatment of MazF to tumor cells. In addition, as both normal and tumor cells are sensitive to MazF, it is quite important to develop a specific and effective delivery and expression system for MazF only in tumor cells. Since co-expression of antitoxin MazE with MazF inhibits mRNA degradation, shutoff of protein synthesis and apoptotic cell death induced by MazF, MazE can be used to confer protection from MazF toxicity in non-tumor cells.

Recently, the TA toxins are being exploited for possible applications in the medical field. For example, we showed that induction of the mazF gene from an HIV-1 promoter leads to degradation of viral mRNAs [Chono et al., 2011; Okamoto et al., 2013]. Results from our laboratory and another group [Park et al., 2012; Shapira et al., 2012] showed that MazF can be used to create novel tools against HIV-1 and hepatitis $C$ viruses. In the future, it is possible to utilize MazF and other bacterial endoribonucleases as tissue-specific and selective anti-cancer agents by using tissue-specific promoters and tissue-specific delivery systems.

\section{Experimental Procedures}

\section{Plasmids}

The MazF and MazE expression plasmids have been described before [Shimazu et al., 2007]. The parent plasmids along with the Tet-repressor expression plasmid, pcDNA6/TR, were purchased from Invitrogen (Carlsbad, Calif., USA).

\section{Cell Lines}

Human embryonic kidney 293 cell lines that stably express MazF alone (293mazF/pcDNA3) and that stably co-express MazE with MazF (293mazF/mazE) were established through stable cotransfection with the pcDNA6/TR and either $\mathrm{pcDNA} 4 / \mathrm{TO} / \mathrm{mazF}$ and pcDNA3 or pcDNA4/TO/mazF and pcDNA3/mazE by PolyFect Transfection Reagent (Qiagen, Inc., Valencia, Calif., USA) according the manufacturer's instructions, and were selected with $5 \mathrm{mg} / \mathrm{ml}$ blasticidin for pcDNA6/TR, $40 \mathrm{mg} / \mathrm{ml}$ zeocin for $\mathrm{pcDNA} 4 / \mathrm{TO} / \mathrm{mazF}$ and $500 \mathrm{mg} / \mathrm{ml}$ geneticin (all Invitrogen).

\section{Cell Culture}

All cell lines were cultured in Dulbecco modified Eagle's media (Gibco, Carlsbad, Calif., USA) including 10\% fetal bovine serum, $0.5 \mathrm{mg} / \mathrm{ml}$ blasticidin, $4 \mathrm{mg} / \mathrm{ml}$ zeocin and $50 \mathrm{mg} / \mathrm{ml}$ geneticin at $37^{\circ} \mathrm{C}$ in $5 \% \mathrm{CO}_{2}$ incubator.

\section{Tumor Formation in Nude Mice}

Tumor formation in nude mice was performed as previously described [Degenhardt et al., 2002]. Cells were harvested by trypsinization and washed twice with sterile phosphate-buffered saline (PBS), and viable cell number was counted by trypan blue exclusion. Cells were diluted in PBS and injected subcutaneously at $1 \times$ $10^{6}$ cells per 6-week-old nude mice (Taconic, Hudson, N.Y., USA). Tumor growth was monitored twice a week by a 6-in dial caliper, beginning at 1 week after injection. To induce MazF, $100 \mu \mathrm{g} / \mathrm{ml}$ Dox (Sigma, St. Louis, Mo., USA) containing sterile water was orally administrated. Tumor-bearing mice were sacrificed and the tumors were excised under sterile conditions for RNA preparation.

\section{$R N A$ Isolation and RT-PCR}

Total RNAs isolated by RNeasy Kit (Qiagen, Inc.) from tumors were subjected to RT-PCR using iScript cDNA Synthesis Kit (BioRad, Hercules, Calif., USA) and specific primers to detect MazF cDNA. Primer sequences are as follows: $5^{\prime}$-ATGGTAAGCCGATACGTACCCGAT- $3^{\prime}$ as forward primer and $5^{\prime}$-CTACCCAATCAGTACGTTAATTTT- $3^{\prime}$ as reverse primer. DNA sequence of MazF cDNA was confirmed.

\section{Disclosure Statement}

Dr. Tsutomu Shimazu, who was a post-doctoral fellow in Dr. Inouye's laboratory, passed away after completion of this work. He had approved the manuscript. Dr. Inouye assumes all the responsibility for this data. 


\section{References}

-Aizenman E, Engelberg-Kulka H, Glaser G: An Escherichia coli chromosomal 'addiction module' regulated by guanosine $3^{\prime}, 5^{\prime}$-bispyrophosphate: a model for programmed bacterial cell death. Proc Natl Acad Sci USA 1996; 93:6059-6063.

Ardelt W, Shogen K, Darzynkiewicz Z: Onconase and amphinase, the antitumor ribonucleases from Rana pipiens oocytes. Curr Pharm Biotechnol 2008;9:215-225.

Arnold U, Ulbrich-Hofmann R: Natural and engineered ribonucleases as potential cancer therapeutics. Biotechnol Lett 2006;28:16151622.

Benito A, Ribo M, Vilanova M: On the track of antitumour ribonucleases. Mol Biosyst 2005; 1:294-302.

-Chono H, Matsumoto K, Tsuda H, Saito N, Lee K, Kim S, Shibata H, Ageyama N, Terao K, Yasutomi Y, Mineno J, Inouye M, Kato I: Acquisition of HIV-1 resistance in T lymphocytes using an ACA-specific E. coli mRNA interferase. Hum Gene Ther 2011;22:35-43.

-Costanzi J, Sidransky D, Navon A, Goldsweig H: Ribonucleases as a novel pro-apoptotic anticancer strategy: review of the preclinical and clinical data for ranpirnase. Cancer Invest 2005;23:643-650
Degenhardt K, Chen G, Lindsten T, White E: BAX and BAK mediate p53-independent suppression of tumorigenesis. Cancer Cell 2002;2: 193-203.

Gerdes K, Christensen, SK, Lobner-Olesen A: Prokaryotic toxin-antitoxin stress response loci. Nat Rev Microbiol 2005;3:371-382.

Inouye M: The discovery of mRNA interferases: implication in bacterial physiology and application to biotechnology. J Cell Physiol 2006; 209:670-676.

Lee I, Shogen K: Mechanisms of enhanced tumoricidal efficacy of multiple small dosages of ranpirnase, the novel cytotoxic ribonuclease, on lung cancer. Cancer Chemother Pharmacol 2008;62:337-346.

Lee JE, Raines RT: Ribonucleases as novel chemotherapeutics: the ranpirnase example. BioDrugs 2008;22:53-58.

Leland PA, Raines RT: Cancer chemotherapy ribonucleases to the rescue. Chem Biol 2001; 8:405-413.

Li GY, Zhang Y, Chan MC, Mal TK, Hoeflich KP, Inouye M, Ikura M: Characterization of dual substrate binding sites in the homodimeric structure of Escherichia coli mRNA interferase MazF. J Mol Biol 2006;357:139-150.

Okamoto M, Chono H, Kawano Y, Saito N, Tsuda H, Inoue K, Kato I, Mineno J, Baba M: Sustained inhibition of HIV-1 replication by conditional expression of the E. coli-derived endoribonuclease MazF in CD4+ T cells. Hum Gene Ther Methods 2013;24:94-103.
Park JH, Yamaguchi Y, Inouye M: Intramolecular regulation of the sequence-specific mRNA interferase activity of MazF fused to a MazE fragment with a linker cleavable by specific proteases. Appl Environ Microbiol 2012;78: 3794-3799.

-Shapira A, Shapira S, Gal-Tanamy M, Zemel R, Tur-Kaspa R, Benhar I: Removal of hepatitis C virus-infected cells by a zymogenized bacterial toxin. PLoS One 2012; 7:e32320.

-Shimazu T, Degenhardt K, Nur EKA, Zhang J, Yoshida T, Zhang Y, Mathew R, White E, Inouye $\mathrm{M}$ : NBK/BIK antagonizes MCL-1 and BCL-XL and activates BAK-mediated apoptosis in response to protein synthesis inhibition. Genes Dev 2007;21:929-941.

-Wu Y, Mikulski SM, Ardelt W, Rybak SM, Youle RJ: A cytotoxic ribonuclease. Study of the mechanism of onconase cytotoxicity. J Biol Chem 1993;268:10686-10693.

Yamaguchi Y, Park JH, Inouye M: Toxin-antitoxin systems in bacteria and archaea. Ann Rev Genet 2011;45:61-79.

Zhang Y, Zhang J, Hoeflich KP, Ikura M, Qing G, Inouye M: MazF cleaves cellular mRNAs specifically at ACA to block protein synthesis in Escherichia coli. Mol Cell 2003;12:913-923. 